Article

\title{
A Kriging Surrogate Model for the Interference Reduction in the Settlement Surveillance Sensors of Steel Transmission Towers
}

\author{
Jiajia Shi ${ }^{1}\left(\mathbb{D}\right.$, Liu Chu ${ }^{1, *(\mathbb{D})}$ and Eduardo Souza de Cursi ${ }^{2}$ \\ 1 School of Transportation and Civil engineering, Nantong University, Nantong 226019, Jiangsu, China \\ 2 Département Mécanique, Institut National des Sciences Appliquées de Rouen, \\ 76800 Saint-Étienne-du-Rouvray, France \\ * Correspondence: chuliu@ntu.edu.cn
}

Received: 12 June 2019; Accepted: 9 August 2019; Published: 14 August 2019

\begin{abstract}
The utilization of modal frequency sensors is a feasible and effective way to monitor the settlement problem of the transmission tower foundation. However, the uncertainties and interference in the real operation environment of transmission towers highly affect the accuracy and identification of modal frequency sensors. In order to reduce the interference of modal frequency sensors for transmission towers, a Kriging surrogate model is proposed in this study. The finite element model of typical transmission towers is created and validated to provide the effective original database for the Kriging surrogate model. The prediction accuracy and convergences of the Kriging surrogate model are measured and confirmed. Besides the merits in computational cost and high-efficiency, the Kriging surrogate model is proven to have a satisfied and robust interference reduction capacity. Therefore, the Kriging surrogate model is feasible and competitive for interference filtration in the settlement surveillance sensors of steel transmission towers.
\end{abstract}

Keywords: Kriging surrogate model; surveillance sensor; interference reduction; transmission tower

\section{Introduction}

The steel transmission tower is a significant component of the electrical systems for the power transmission and distribution network [1,2]. The reliability and safety of steel transmission towers are crucial issues in both the design process and the operation periods [3]. The failure or damage of steel transmission towers can cause huge economic losses and even disasters for both industrial manufacturing and people's daily lives [4]. The surveillance sensors are useful to help monitor the real-time status of transmission towers [5]. By the data of surveillance sensors, it is not only possible to predict the safe service life, but it is also possible to avoid the collapse of transmission towers by the maintenance in advance.

The application of surveillance sensors in the key location of the transmission towers to monitor the real-time status attracts great concerns of engineers and researchers. For example, the inclination sensors are effective at reflecting the stress of the large deformation, but the parameters corresponding to the load balance and the yield failure of the local rods are ignored [6]. In addition, the strain sensors by the resistance strain gauge are sensitive enough to identify the fluctuation of forces, but are very susceptible to electromagnetic interference and are not robust enough to avoid rusting in the measurement circuits [7]. Even though the grating fiber strain sensors have some level of improvement in the measurement accuracy and range, the limitation in the installation location and quantity is not completely overcome [8].

The modal identification sensors are one of the promising technologies and have been widely used in structural health monitoring, such as in the reinforced concrete bridges $[9,10]$ and wind turbine 
blades [11]. By the modal identification of the reinforced concrete bridges, the passing vehicles on the bridge pavement deck and fatigue damage of the bridge body are tracked and recorded. Moreover, the modal frequency sensors are effective in identifying the vibration of wind turbine blade and are sensitive to the structural changes related to damage or failures. Furthermore, the modal identification technology is also applied in the safety and reliability analysis of transmission towers [12].

The inevitable uncertainties and interference in the modal identification of transmission towers are originated from several aspects. First, the terrain environment often has uncontrollable factors, such as the ground displacement, foundation inclination and even cracking and subsidence of rocks. In addition, the natural climate and weather environment also provides unpredictable uncertainties [13]. For example, snow in the winter not only evidently increases the weight of the transmission towers, but also heavily influences the material properties of steel, which causes the reduction of structural stiffness and strength and makes transmission towers fragile to fracture and collapse. Besides, the random wind force is non-negligible to affect the modal identification of transmission towers [14,15]. Under the strong winds [16], such as hurricanes, tornados or isolated thunderstorms, the nonlinear inelastic behaviors of transmission tower structures become dominated $[17,18]$. The quasi-steady dynamic loads make the modal frequencies of transmission towers difficult to analyze. Therefore, it is necessary to develop the feasible analytical model to identify and reduce the interference in modal identification sensors of transmission towers.

This paper proposes a Kriging surrogate model to effectively reduce the interference in settlement surveillance sensors of steel transmission towers. At first, the finite element model of the transmission tower structure is created based on the ANSYS Parameter Design Language (ANSYS, APDL, Version 14.5). Meanwhile, the first five order vibration modes and natural frequencies are computed and recorded. The verification for the numerical simulation model of the transmission towers is performed by comparing it with the data from the experimental measurements. Next, based on the database of natural frequencies computed by the finite element method, a Kriging surrogate model is built to effectively reduce the interference or uncertainties in the identification of the transmission tower settlement. The accuracy and convergence of the Kriging surrogate model are confirmed in typical tests. Finally, some brief summaries are drawn in the last section.

\section{Problem Formulation and Theoretical Methods}

\subsection{Steel Transmission Towers}

The structures of transmission towers have multiple types. The original finite element model of the transmission tower structure in this study is presented in Figure 1. The jointed trusses in the finite element model of the transmission tower are meshed with the beam finite elements. There are 690 trusses, 2524 beam elements and 2168 nodes.

In addition, the parameters corresponding to the geometrical properties of the transmission tower are shown in Figure 1, and the serial number for the transmission tower is 2A2-J1. For the material property parameters, the Young's modulus is $2 \times 10^{11} \mathrm{~Pa}$, the physical density is $7850 \mathrm{~kg} / \mathrm{m}^{3}$ and the Poisson ratio is 0.28 . The fluctuations and uncertainties in the material and geometrical property parameters caused by the environment, weather, operation status, etc., are supposed to be the interference in the settlement surveillance sensor.

The relationship between the parameters corresponding with the settlement in the transmission tower and the natural frequencies is implicit. Creating the explicit function to express the mathematical relationship is not feasible in the safety surveillance of transmission towers. By using the Kriging surrogate model, the implicit expression of the parameters is successfully represented, and the input signals provided by the sensors installed in the transmission tower can be effectively filtered for settlement identification. 

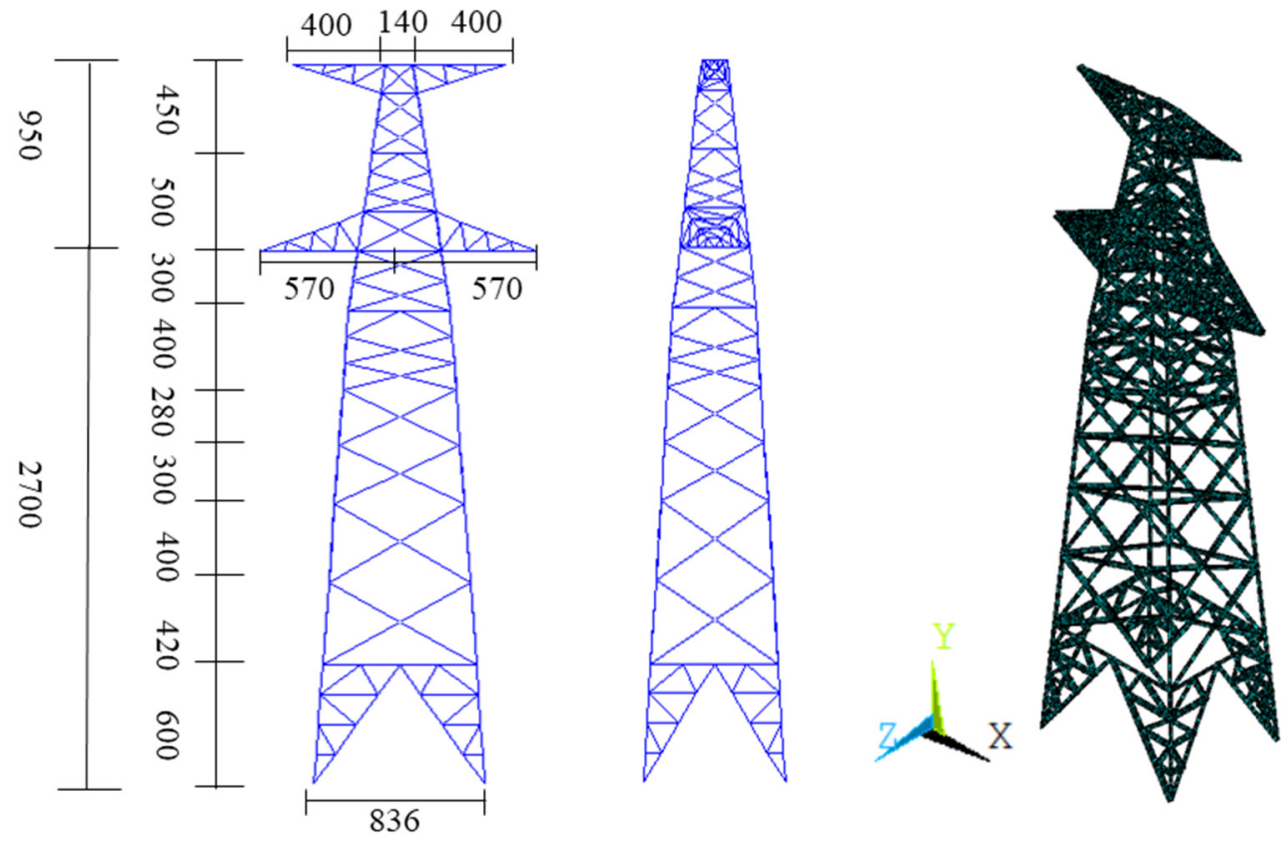

Figure 1. The finite element model of the transmission tower structure (the unit is $\mathrm{cm}$, serial number [19] for the transmission tower is 2A2-J1).

\subsection{Kriging Surrogate Model}

The Kriging surrogate model is a stochastic interpolation algorithm that assumes that the model output is a realization of a Gaussian process indexed by $x \in D_{X} \subset \mathbb{R}^{M}$. A Kriging model is described by the following equation $[20,21]$ :

$$
y \approx M^{K}(x)=\beta^{T} f(x)+\sigma^{2} Z(x, w)
$$

where $y=M(x)$ is the output data and $\beta^{T} f(x)$ and $\sigma^{2}$ are the mean and variance of the Gaussian process, respectively. $Z(x, w)$ is a zero mean, unit variance, stationary Gaussian process, which is characterized by a correlation function $R$ with hyper-parameters $\theta$.

Given $\mathbf{X}=\left\{x_{1}, x_{2}, \cdots, x_{n}\right\}$, then $\mathbf{y}=\left\{M^{K}\left(x_{1}\right), M^{K}\left(x_{2}\right), \cdots, M^{K}\left(x_{n}\right)\right\}^{T}$, and the calculation of prediction $M^{K}(x)$ in the point $x$ is based on the Gaussian properties of the process.

The Gaussian assumption states that the vector formed by the true model responses, $\mathbf{y}$ and the prediction, $\hat{\mathbf{Y}}(x)$ has a joint Gaussian distribution defined by [22]:

$$
\left\{\begin{array}{c}
\hat{\mathbf{Y}}(x) \\
\mathbf{y}
\end{array}\right\} \sim N_{N+1}\left(\left\{\begin{array}{c}
f^{T}(x) \beta \\
F \beta
\end{array}\right\}, \sigma^{2}\left\{\begin{array}{cc}
1 & r^{T}(x) \\
r(x) & R
\end{array}\right\}\right)
$$

where $F$ is the information matrix of generic terms: $F_{i j}=f_{j}\left(x_{i}\right), i=1, \cdots, N, j=1, \cdots, P ; r(x)$ is the vector of cross-correlations between the prediction point $x$ and each one of the observations whose terms read: $\mathbf{r}_{i}=R\left(x, x_{i} ; \theta\right), i=1, \cdots, N$ and $R$ is the correlation matrix whose terms are written as:

$$
R_{i j}=R\left(x, x_{i} ; \theta\right), i, j=1, \cdots, N .
$$

Consequently, the mean and variance of the Gaussian random variate $\hat{\mathbf{Y}}(x)$ can be calculated as [23]:

$$
\sigma_{\hat{\mathbf{Y}}}^{2}(x)=\sigma^{2}\left(1-r^{T}(x) R^{-1} r(x)+u^{T}(x)\left(F^{T} R^{-1} F\right)^{-1} u(x)\right)
$$




$$
\beta=\left(F^{T} R^{-1} F\right)^{-1} F^{T} R^{-1} y .
$$

The generalized least-square estimate of the underlying regression problem can be expressed as [24]:

$$
u(x)=F^{T} R^{-1} r(x)-f(x) .
$$

Another useful corollary of the Gaussian assumption is that:

$$
\hat{\mathbf{Y}}(x) \sim N\left(\mu_{\hat{\mathbf{Y}}}(x), \sigma_{\hat{\mathbf{Y}}}^{2}(x)\right) .
$$

Thus,

$$
P[\hat{\mathbf{Y}}(x) \leq t]=\phi\left(\frac{t-\mu_{\hat{\mathbf{Y}}}(x)}{\sigma_{\hat{\mathbf{Y}}}(x)}\right)
$$

where $\phi(\cdot)$ denotes the Gaussian cumulative density function. Based on the above equation, the confidence intervals on the predictor can be calculated by Equation (9) with probability $1-\alpha$.

$$
\hat{\mathbf{Y}}(x) \in\left[\mu_{\hat{\mathbf{Y}}}(x)-\phi^{-1}\left(1-\frac{\alpha}{2}\right) \sigma_{\hat{\mathbf{Y}}}(x), \mu_{\hat{\mathbf{Y}}}(x)+\phi^{-1}\left(1-\frac{\alpha}{2}\right) \sigma_{\hat{\mathbf{Y}}}(x)\right] .
$$

In order to create the Kriging surrogate model and perform it to provide precise prediction, the functional basis of the Kriging trend should be selected first, and then the appropriate correlation function $R\left(x, x^{\prime} ; \theta\right)$ is chosen. If the hyper-parameters $\theta$ and the Gaussian process variance $\sigma^{2}$ are unknown, then this involves setting up an optimization problem and solving it. Using the optimal value of $\theta$, the rest of the unknown Kriging parameters $\left(\sigma^{2}, \beta\right)$ can be calculated.

\subsection{Interference Reduction in Settlement Surveillance Sensor}

The interference reduction in the settlement surveillance sensor strongly depends on the original database of the finite element computation for the natural frequencies of the transmission tower structure. The parameters corresponding with the settlement of the transmission tower and the natural frequencies are related to the Kriging surrogate model by implicit expression. By the Kriging surrogate model, the input signals provided by the sensors installed in the transmission tower are filtered, and the interference signals are effectively reduced to identify the settlement. Then, real time safety surveillance of the transmission tower is successfully implemented. The process of interference reduction in the settlement surveillance sensor is shown in Figure 2.

Due to the satisfied accuracy and feasibility, and the computational cost saving compared with the finite element method, the Kriging surrogate model is chosen for interference reduction in the settlement surveillance sensor. The uncertainty and randomness of the settlement in the transmission tower are propagated as that in the real situation. A number of 1000 stochastic samples of the transmission tower with the settlement are simulated by the finite element model. The records of the transmission tower settlement and the corresponding natural frequencies in the five vibration modes are shown in Figure 3. The results of the natural frequencies and related settlement of transmission tower provide the useful original database for the Kriging surrogate model. 


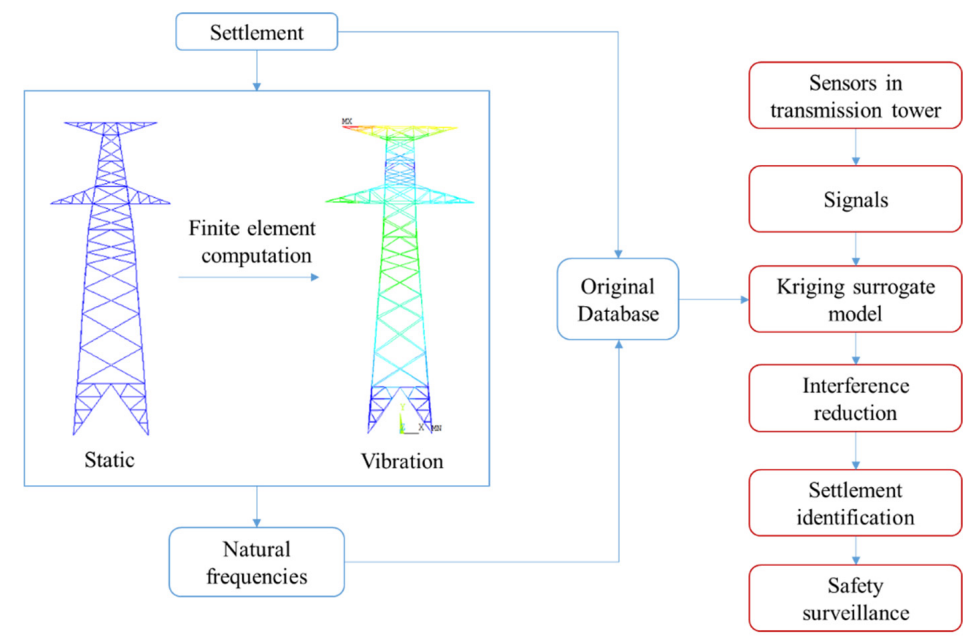

Figure 2. Schematic diagram of interference reduction in the settlement surveillance sensor.

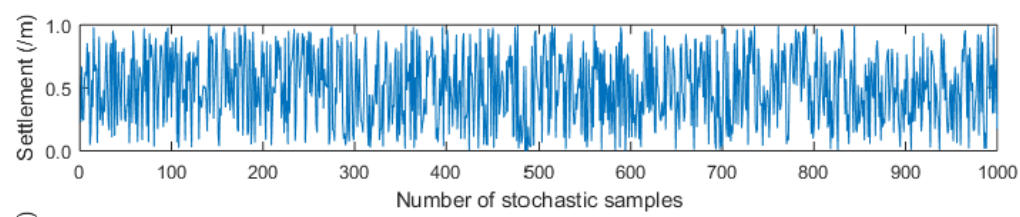

(a)

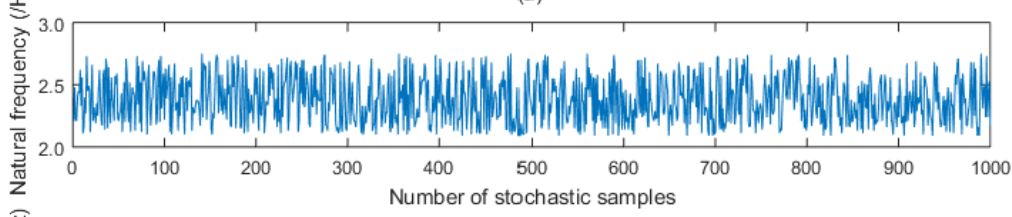

$\stackrel{\widehat{N}}{\underline{T}}$

(b)
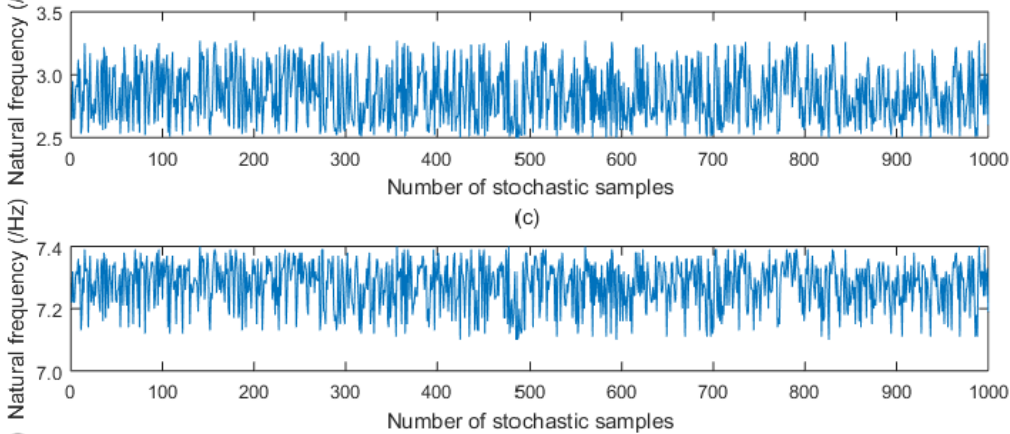

(d)
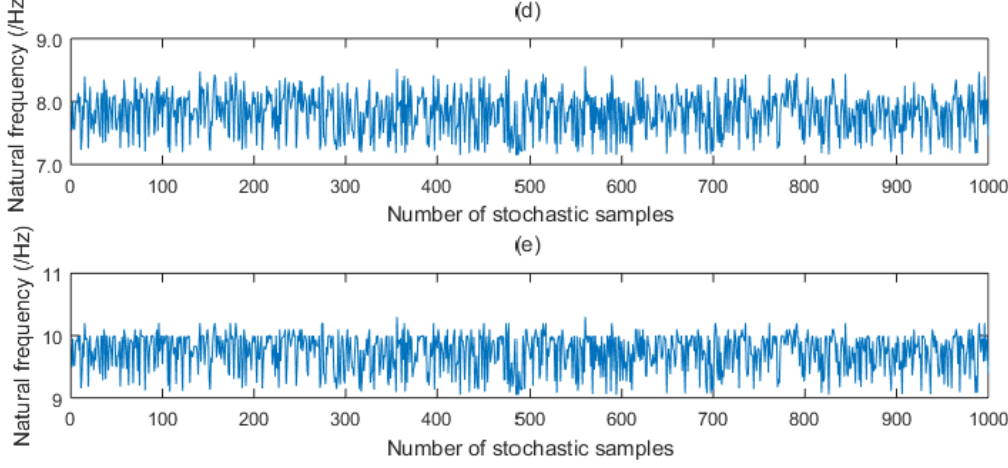

(f)

Figure 3. Records of the transmission tower settlement and the corresponding natural frequencies (a) is the records of the transmission tower settlement, and $(\mathbf{b}-\mathbf{f})$ are the natural frequency records of the first to fifth order vibration modes, respectively. 


\section{Results and Discussion}

\section{Validation of Finite Element Model}

For the validation of the transmission tower finite element model, the displacement and rotation of the first order vibration mode are computed and presented in Figures 4 and 5. The $\mathrm{X}, \mathrm{Y}$ and $\mathrm{Z}$ directions in the finite element model of the transmission tower are marked in Figure 1. In Figure 4, the displacement in the $\mathrm{Z}$ direction is more primary than that in the $\mathrm{X}$ and $\mathrm{Y}$ direction. However, the rotation in the $X$ direction is more substantial and closer to the sum vector result in Figure 5. The results demonstrated in Figures 4 and 5 are quite true to the facts.

In addition, the displacement and rotation results of the transmission tower with settlement are computed by the finite element method and revealed in Figures 6 and 7. Different from the transmission tower without settlement, the settlement makes the natural frequencies of the transmission tower obviously amplified. Furthermore, even though the displacement in the $\mathrm{Z}$ direction plays an essential role in the sum vector result, the changes of the displacement in the X direction are evident in Figure 6 when compared with that in Figure 4. Besides, the most dangerous locations in the displacement in the $\mathrm{X}$ direction move up in Figure 6a when the settlement happens. The modifications in the rotation of the transmission tower caused by settlement are apparent in the $\mathrm{Y}$ and $\mathrm{Z}$ directions, as shown in Figure 7. The rotation in the $X$ direction is more dominant in the sum vector result, as shown in Figure 5. The experimental test and numerical simulation in the reported literature [5] also prove that the $X$ direction is most sensitive in detecting the settlement of the transmission tower. The displacement of the transmission tower in the $X$ direction is obviously fluctuated after the settlement and can be cited as the effective evidence for the identification and judgment of the foundation settlement. Thus, the finite element model of the transmission towers is verified to analyze the settlement by the natural frequencies and vibration modes.

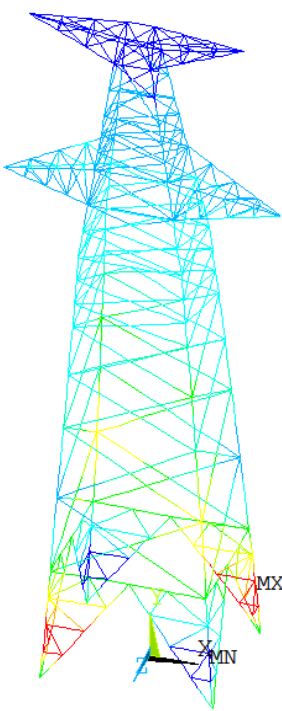

(a)

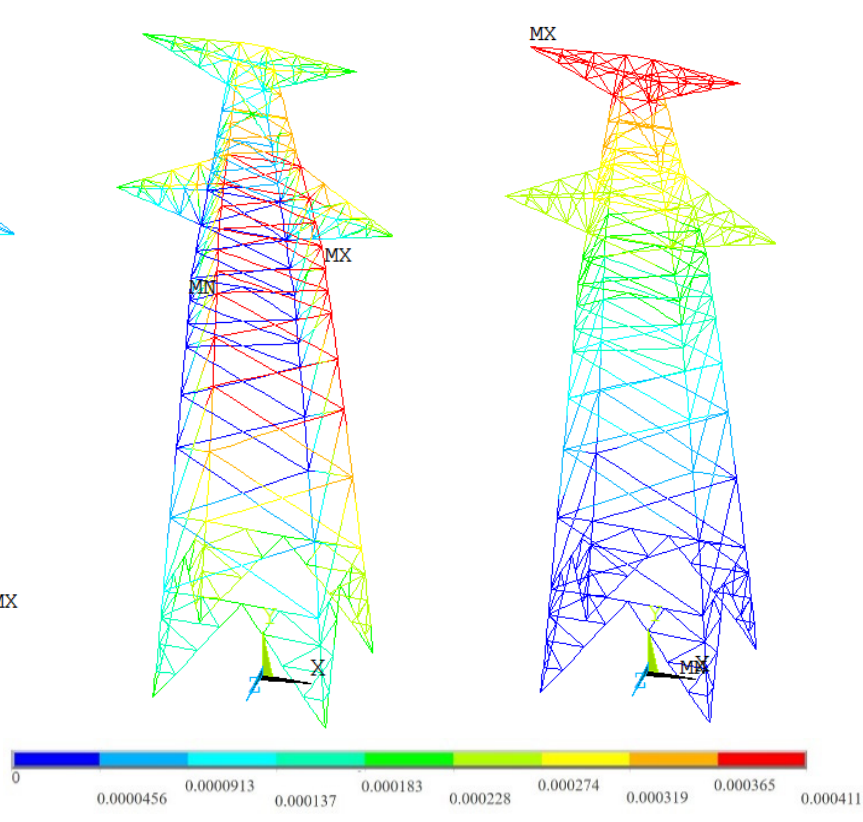

(b)

(c)

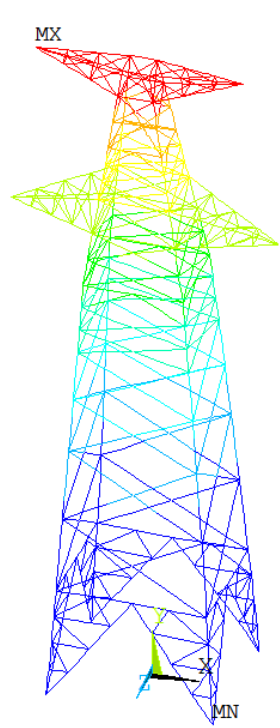

(d)

Figure 4. Displacement of the transmission tower in the first order vibration mode; $(\mathbf{a}-\mathbf{c})$ are the results in the $\mathrm{X}, \mathrm{Y}$ and $\mathrm{Z}$ directions, respectively, and (d) is the sum vector result. 


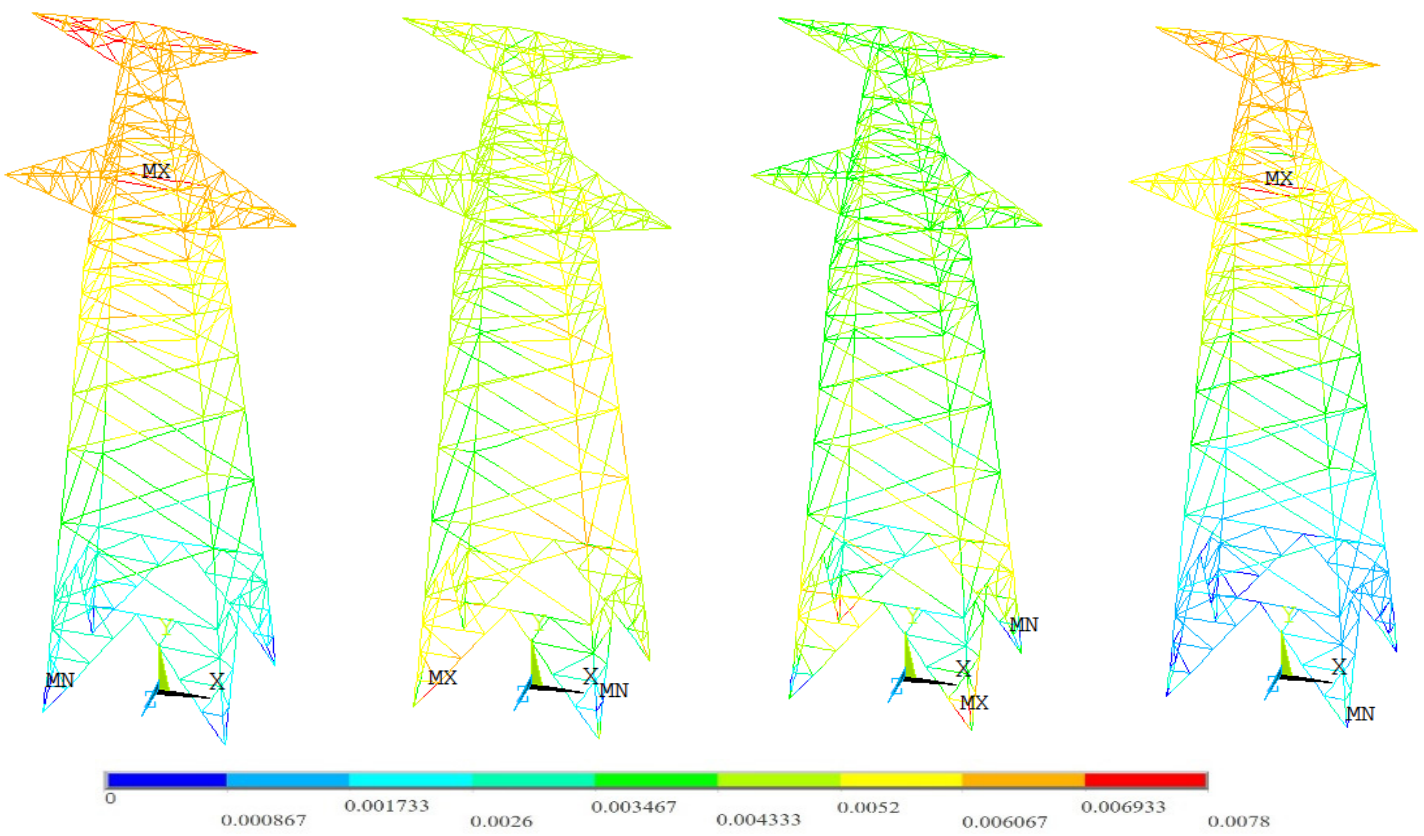

(a)

(b)

(c)

(d)

Figure 5. Rotation of the transmission tower in the first order vibration mode; $(\mathbf{a}-\mathbf{c})$ are the results in the $\mathrm{X}, \mathrm{Y}$ and $\mathrm{Z}$ directions, respectively, and (d) is the sum vector result.
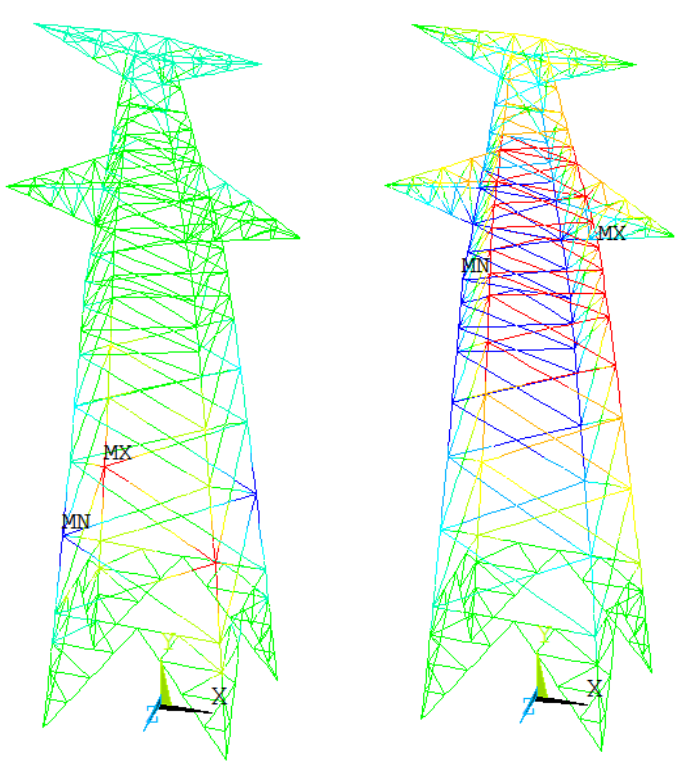

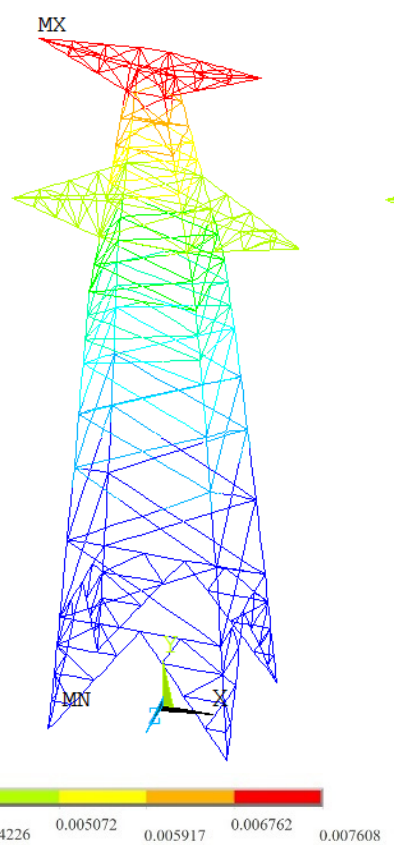

(c)

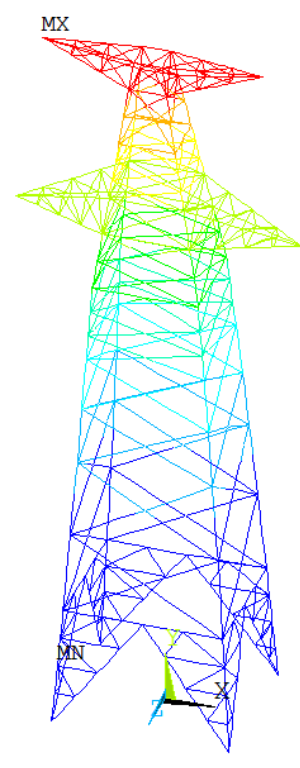

(d)

Figure 6. Displacement of the transmission tower in the first order vibration mode with settlement; $(\mathbf{a}-\mathbf{c})$ are the results in the $\mathrm{X}, \mathrm{Y}$ and $\mathrm{Z}$ directions, respectively, and (d) is the sum vector result. 


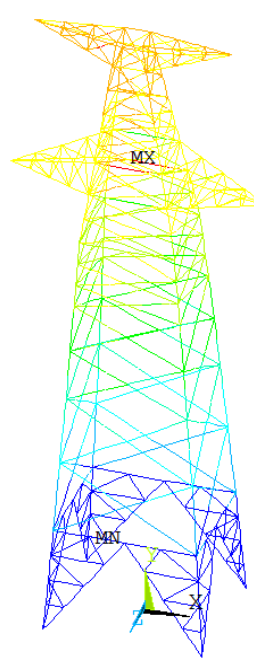

(a)

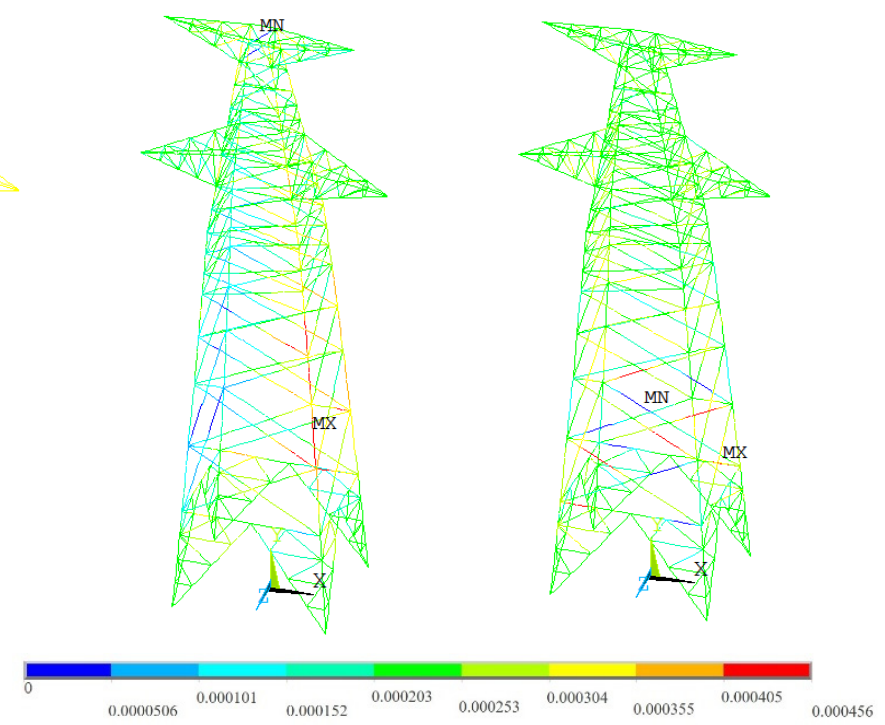

(b)

(c)

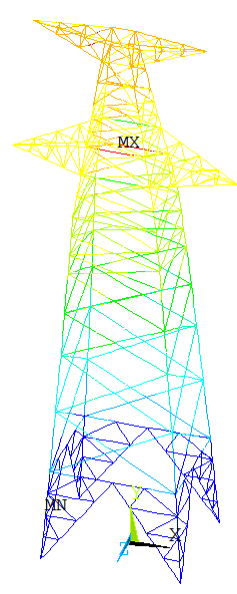

(d)

Figure 7. Rotation of the transmission tower in the first order vibration mode with settlement; $(\mathbf{a}-\mathbf{c})$ are for the $\mathrm{X}, \mathrm{Y}$ and $\mathrm{Z}$ directions, respectively, and (d) is for the sum vector result.

\section{Settlement Identification}

As explained by the schematic diagram of the interference reduction in the settlement surveillance sensor in Figure 2, the Kriging surrogate model is performed to identify the settlement and effectively reduce the interference in the settlement surveillance sensor. Based on the original database provided by the finite element model computation, the implicit relationship between the natural frequencies and the settlement are effectually created. When the natural frequencies of the transmission tower are measured and recorded by the sensor, the settlement can be predicted and evaluated by the Kriging surrogate model for the safety surveillance. The accuracy of the Kriging surrogate model for the identification of the transmission tower settlement is tested and compared with the precise solution of the finite element model, as shown in Figures 8 and 9.

In Figure 8, the differences between the Kriging surrogate model prediction and the precise results of the transmission tower finite element model for the first five natural frequencies are demonstrated. By comparison, it is obvious that the Kriging surrogate model provides more accurate predictions in the low order vibration modes for transmission towers, as the relative errors in Figure $8 \mathrm{a}, \mathrm{b}$ are evidently smaller than that in Figure 8d,e. In addition, the prediction accuracy of the Kriging surrogate model in the third vibration mode is more satisfied than that of the first two order natural frequencies. The reasons for this phenomenon can be several possibilities. The better original database of finite element results in the local interval can cause more accurate predictions of the Kriging surrogate model in Figure 8c. The further confirmation is performed in the following. Even the differences between the Kriging surrogate model predictions with the precise results have fluctuations, the relative errors are smaller than $0.01 \mathrm{~Hz}$ for natural frequencies in the first two vibration modes. The sharp peak or high plateau is not present in the low vibration modes. The evident oscillation is marked in Figure $8 \mathrm{~d}$,e. The threshold to track the oscillation for the Kriging surrogate model prediction is when the relative errors are continuously larger than $0.005 \mathrm{~Hz}$. Thus, the low orders such as the first and the second natural frequencies are useful to be tracked and recorded as the settlement reference for transmission towers. 

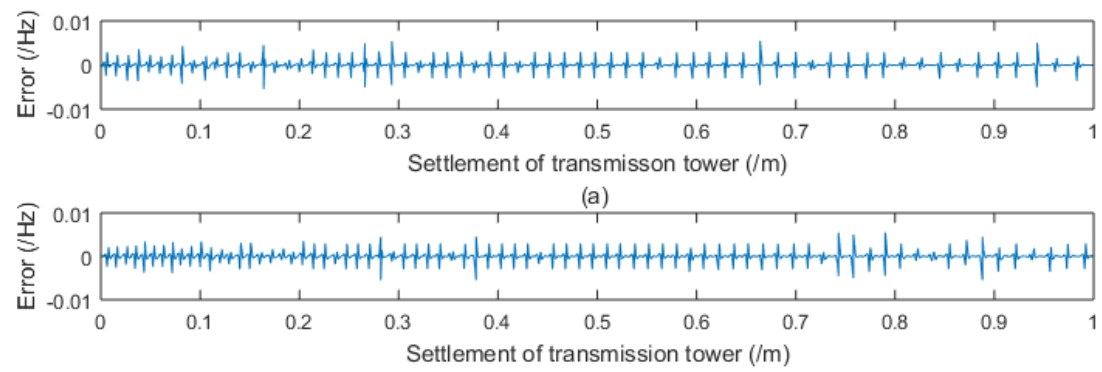
(b)
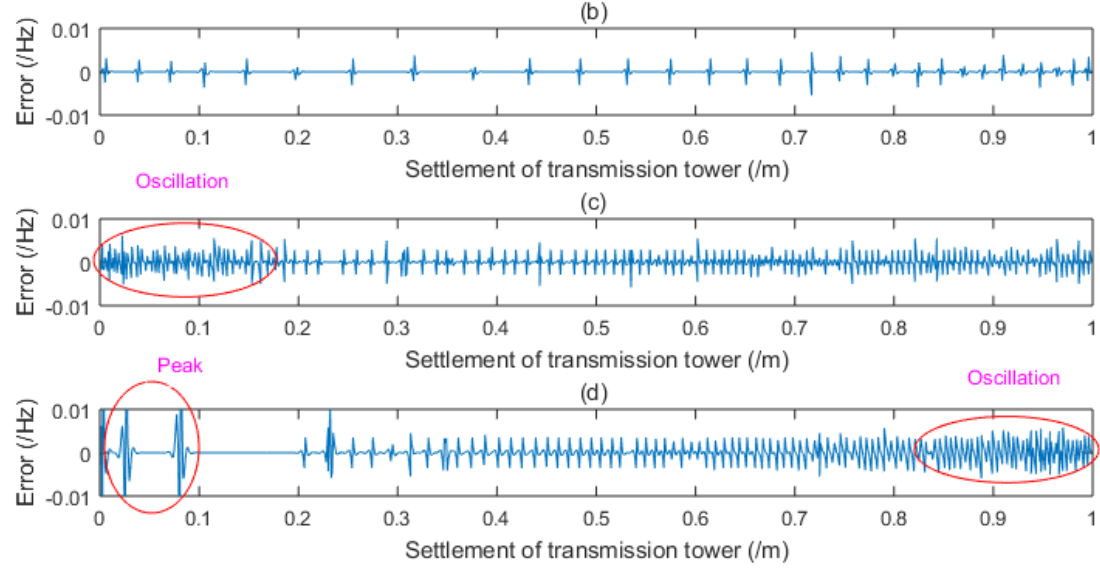

(e)

Figure 8. The differences between the Kriging surrogate model prediction and precise results; (a-e) are for first five vibration modes, respectively.

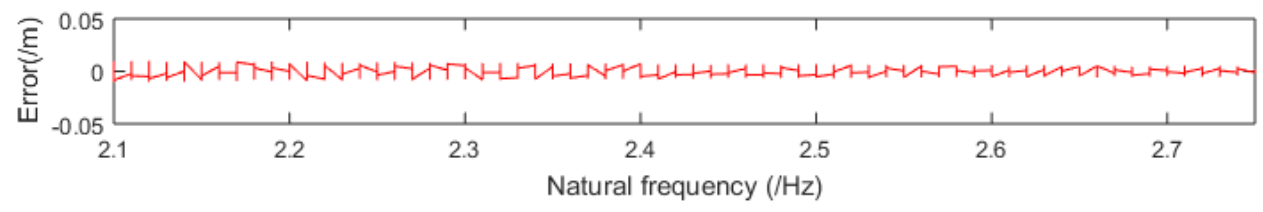

(a)

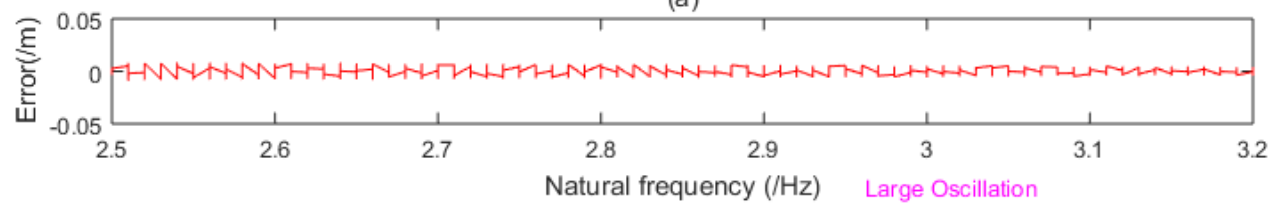

(b)

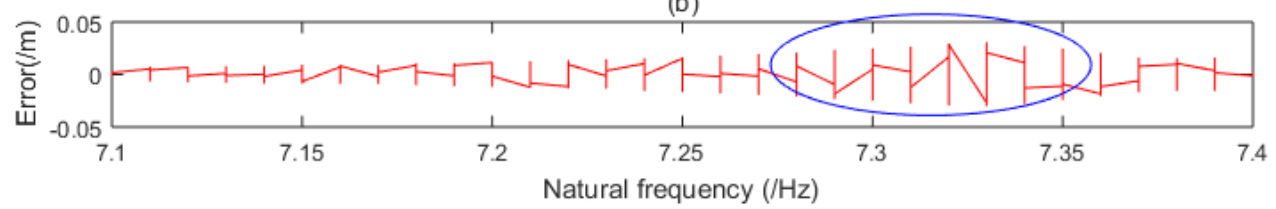

(c)

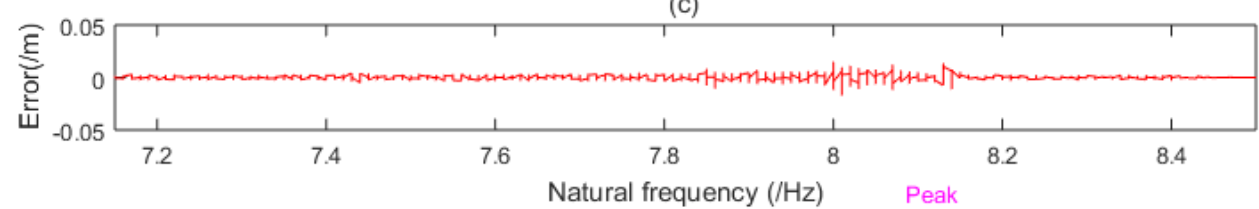

(d)

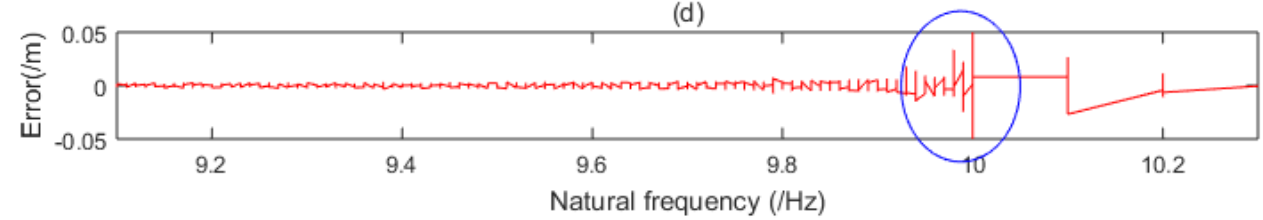

(e)

Figure 9. The settlement identifications of the Kriging surrogate model by the inverse function (a-e) are the errors of the first five vibration modes, respectively. 
To be more comprehensive, the settlement identifications of the Kriging surrogate model by the inverse function are presented in Figure 9. For the normal implicit function of the Kriging surrogate model, the input information is the settlement possibly happened in the transmission tower, while the output results are the natural frequencies predicted by the Kriging surrogate model. On the contrary, for the inverse implicit function, the input database of the Kriging surrogate model is the natural frequencies of the transmission tower, while the predictive results are the settlement of foundation. Based on the available natural frequencies tested and recorded by the surveillance sensor, the settlement of the transmission tower is calculated by the Kriging surrogate model.

As shown in Figure 9a,b, the settlement identification of the Kriging surrogate model is satisfied in the first two vibration modes. However, different from the results in Figure 8c, the merits of the Kriging surrogate model in the third vibration mode disappear in Figure $9 \mathrm{~d}$ for settlement identification. Different from the threshold in Figure 9, the oscillation for the settlement results for the transmission tower is marked with the red circle when the value of the relative error is larger than $0.025 \mathrm{~m}$. In addition, even the Kriging surrogate model offers better results based on the high order natural frequencies in the local intervals, and the instability in Figure $9 \mathrm{e}$ is unneglectable. The reasons for the selection of the low order natural frequencies to identify the settlement of transmission tower include two aspects. On the one hand, the natural frequencies of low order vibration modes before and after settlement are obvious both in experiments and numerical simulation. On the other hand, the instability and fluctuation in the natural frequencies of high order vibration modes make the settlement identification lack accurate and convergent results. Therefore, compared with the high order natural frequencies, the first two order natural frequencies provided by the surveillance sensor are more robust to the settlement prediction of the Kriging surrogate model.

\section{Interference Reduction}

The first two order natural frequencies of the transmission tower are verified to be effective references recorded by the surveillance sensor for settlement identification. The capacity of interference reduction in the Kriging surrogate model is measured by taking the uncertainties of the material property parameters into consideration.

According to the real operation situation of transmission towers, the uncertainties and fluctuations in the material parameters, such as the Young's modulus, Poisson's ratio and physical density, are propagated in the finite element model. The signals received by the settlement surveillance sensor are interfered with by the factors corresponding to the changes and uncertainties in the material properties of transmission towers. The Kriging surrogate model is used to identify and filter the interference. The filtration percentage discussed in this study is the percentage of uncertain interference identified by the Kriging surrogate model for the transmission tower. The calculation of filtration percentage is based on the statistical mathematics. According to the two different order natural frequencies of the transmission tower, the prediction results of the settlement by the Kriging surrogate model form the sampling space for the interference identification. The predictive results of the Kriging surrogate model present the discrepancy or fluctuation if there are interferences and uncertainties in the system. The settlement discrepancy between two order natural frequencies is tracked and recorded for interference filtration.

By the Kriging surrogate model proposed in this study, the filtration percentage results of interference reduction for the settlement surveillance sensor are presented in Figure 10. Each subfigure selects two order natural frequencies to jointly calculate the filtration percentage. For example, F1-F2 means the filtration percentage when taking the first and second order natural frequencies into consideration. Firstly, in Figure 10b-d, it can be found that the filtration percentages by the Kriging surrogate model are very stable and satisfied, and the values stay above $92 \%$ even if the accuracy of the Kriging surrogate model decreases. Thus, the first and the third order (F1-F3), the first and the fourth order (F1-F4) and the first and the fifth order (F1-F4) natural frequencies are the satisfied and robust signals used to identify and filter the interference in the settlement surveillance sensor. 
In addition, the second and the third order (F2-F3), the second and the fourth order (F2-F4) and the second and the fifth order (F2-F4) natural frequencies, as shown in Figure 10e-g, can also reach as high as $92 \%$ of interference reduction, but with small performance degradation along with the accuracy decline of the Kriging surrogate model. The third and the fourth order (F3-F4) natural frequencies offered by the settlement surveillance sensor are not decent references to identify or judge the interference. Finally, according to the results in Figure 10a,i,j, with the accuracy reduction of the Kriging surrogate model, the filtration percentage results have evident deterioration in the settlement surveillance sensor. However, the Kriging surrogate model still provides $90 \%$ of filtration percentage in the cases of Figure 10a,i,j. Therefore, the Kriging surrogate model provides a satisfied and robust interference filtration capacity for the settlement surveillance sensor of the transmission tower.

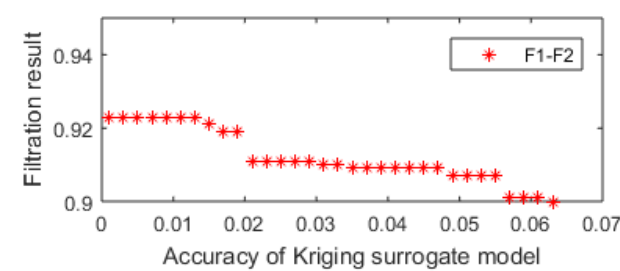

(a)

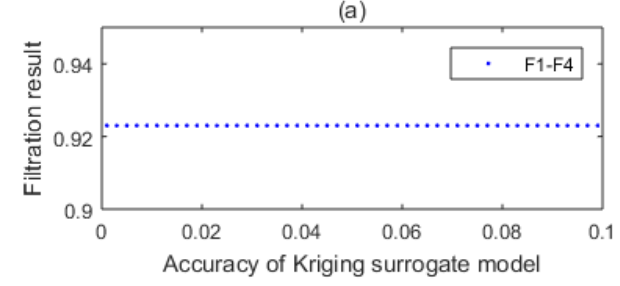

(c)

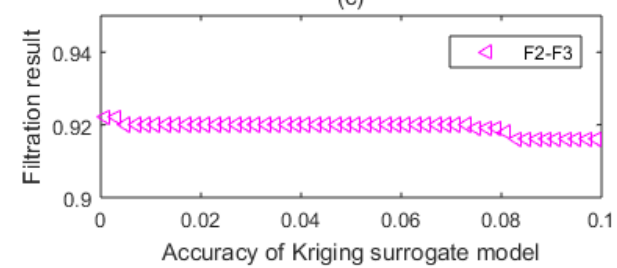

(e)

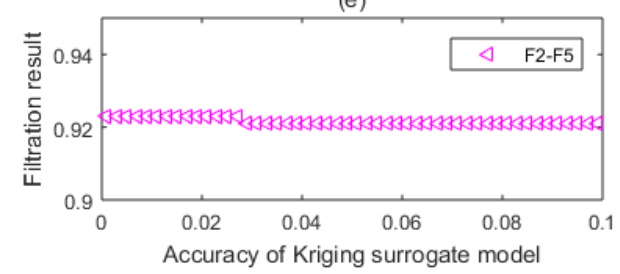

(g)

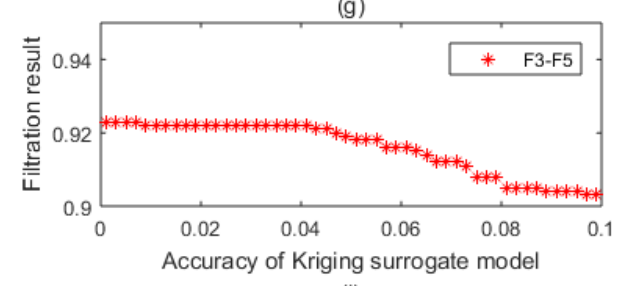

(i)

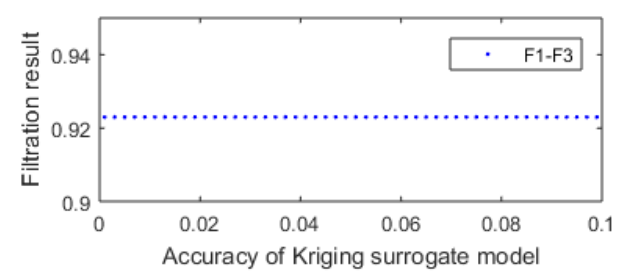

(b)

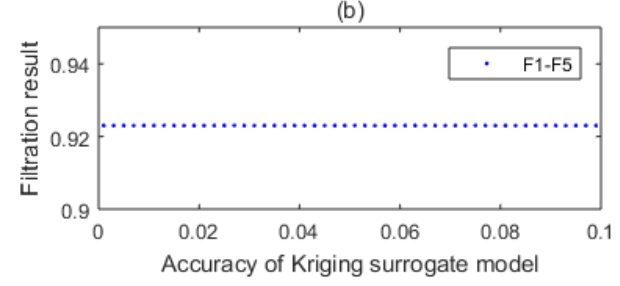

(d)
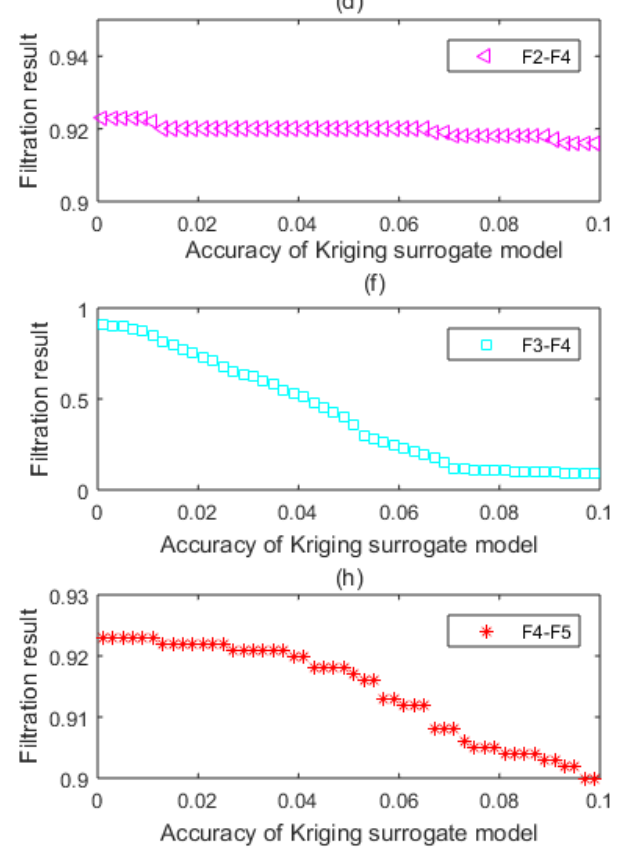

(j)

Figure 10. Filtration percentage results of interference reduction by the Kriging surrogate model for the settlement surveillance sensor (F1, F2, F3, F4 and F5 are the first five order natural frequencies, respectively). (a): F1-F2; (b): F1-F3; (c): F1-F4; (d): F1-F5; (e): F2-F3; (f): F2-F4; (g): F2-F5; (h): F3-F4; (i): F3-F5; (j): F4-F5.

Furthermore, the Kriging surrogate model also has time-saving and high-efficiency merits in settlement surveillance sensors. The finite element model for natural frequency computation takes more time, is expensive and lacks the inverse judgment ability to identify the complicated interference. 
Besides, the prediction and identification for the 5000 sample signals of natural frequencies form the settlement surveillance sensor only cost $20.44 \mathrm{~s}$. The proposed Kriging surrogate model is to be embedded in the safety surveillance system of transmission tower for interference reduction in the settlement identification, instead of replacing the finite element model. The data of natural frequencies of the transmission tower in the real system are collected and transferred by modal identification sensor rather than from the finite element model in the simulation. Furthermore, the rapid sample treatment speed and high computational efficiency also make the Kriging surrogate model very competitive in modal identification sensors. Therefore, the Kriging surrogate model not only provides the satisfied prediction accuracy and robust interference filtration, but also is very competitive in computational costs.

\section{Conclusions}

In this paper, a Kriging surrogate model is proposed to effectively reduce the interference in the settlement surveillance sensor for the transmission tower. According to the comparison and analysis, the results show that:

1. The displacement of transmission towers in the $X$ direction is obviously fluctuated after the settlement and can be cited as the effective evidence for the foundation settlement.

2. Based on the original database of the finite element model of transmission towers, the Kriging surrogate model provides accurate prediction for the natural frequencies and settlement.

3. Compared with the high order natural frequencies, the first two order natural frequencies provided by the surveillance sensor are more robust for the settlement prediction by the Kriging surrogate model.

4. The Kriging surrogate model performs the satisfied and robust interference filtration as high as $92 \%$ for the settlement surveillance sensor of the transmission tower.

5. The merits of computational costs and high efficiency in the Kriging surrogate model make this model feasible and competitive in settlement surveillance sensors.

Author Contributions: Conceptualization, J.S. and L.C.; methodology, L.C.; software, L.C.; validation, J.S. and L.C.; formal analysis, J.S and L.C.; investigation, L.C.; resources, E.S.d.C; data curation, L.C.; writing—original draft preparation, J.S.; writing - review and editing, L.C.; visualization, J.S; supervision, E.S.d.C.; project administration, E.S.d.C.; funding acquisition, E.S.d.C.

Funding: This research was funded by the National Natural Science Foundation of China Grant Nos. 61801247 and 61901235 .

Conflicts of Interest: The authors declare no conflicts of interest.

\section{References}

1. Couceiro, I.; Paris, J.; Martinez, S.; Colominas, I.; Navarrina, F.; Casteleiro, M. Structural optimization of lattice steel transmission towers. Eng. Struct. 2016, 117, 274-286. [CrossRef]

2. Guo, H.Y.; Li, Z.L. Structural topology optimization of high-voltage transmission tower with discrete variables. Struct. Multidiscip. Optim. 2011, 43, 851-861. [CrossRef]

3. Li, X.; Zhang, W.; Niu, H.; Wu, Z.Y. Probabilistic capacity assessment of single circuit transmission tower-line system subjected to strong winds. Eng. Struct. 2018, 175, 517-530. [CrossRef]

4. Savory, E.; Parke, G.A.; Zeinoddini, M.; Toy, N.; Disney, P. Modelling of tornado and microburst-induced wind loading and failure of a lattice transmission tower. Eng. Struct. 2001, 23, 365-375. [CrossRef]

5. Huang, X.; Zhao, Y.; Zhao, L.; Yang, L. A Method for Settlement Detection of the Transmission Line Tower under Wind Force. Sensors 2018, 18, 4355. [CrossRef] [PubMed]

6. Malhara, S.; Vittal, V. Mechanical State Estimation of Overhead Transmission Lines Using Tilt Sensors. IEEE Trans. Power Syst. 2010, 25, 1282-1290. [CrossRef]

7. Xia, Y.; Zhang, P.; Ni, Y.-Q.; Zhu, H.-P. Deformation monitoring of a super-tall structure using real-time strain data. Eng. Struct. 2014, 67, 29-38. [CrossRef] 
8. Bang, H.-J.; Kim, H.-I.; Lee, K.-S. Measurement of strain and bending deflection of a wind turbine tower using arrayed FBG sensors. Int. J. Precis. Eng. Manuf. 2012, 13, 2121-2126. [CrossRef]

9. Huynh, T.-C.; Park, J.-H.; Kim, J.-T. Structural identification of cable-stayed bridge under back-to-back typhoons by wireless vibration monitoring. Measurement 2016, 88, 385-401. [CrossRef]

10. Ding, K. Application of Wavelet Analysis of Curvature Modal to Damage Detection of Bridges. Noise Vib. Control 2013, 33, 131-135.

11. Ou, Y.W.; Eleni, N.C.; Vasilis, K.D.; Minas, D.S. Vibration-based experimental damage detection of a small-scale wind turbine blade. Struct. Health Monit. 2017, 16, 79-96. [CrossRef]

12. Iwaniec, J.; Iwaniec, M.; Kasprzyk, S. Transverse vibrations of transmission tower of variable geometrical structure. J. Low Freq. Noise Vib. Act. Control. 2019, 38. [CrossRef]

13. El Damatty, A.; Elawady, A. Critical load cases for lattice transmission line structures subjected to downbursts: Economic implications for design of transmission lines. Eng. Struct. 2018, 159, 213-226. [CrossRef]

14. Cai, Y.; Xie, Q.; Xue, S.; Hu, L.; Kareem, A. Fragility modelling framework for transmission line towers under winds. Eng. Struct. 2019, 191, 686-697. [CrossRef]

15. Abd-Elaal, E.-S.; Mills, J.E.; Ma, X. A review of transmission line systems under downburst wind loads. J. Wind. Eng. Ind. Aerodyn. 2018, 179, 503-513. [CrossRef]

16. Hamzah, N.H.; Usman, F. Geospatial analysis of wind velocity to determine wind loading on transmission tower. Wind Struct. 2019, 28, 381-388.

17. Zhou, Q.; Zhang, H.; Ma, B.; Huang, Y. Wind loads on transmission tower bodies under skew winds with both yaw and tilt angles. J. Wind. Eng. Ind. Aerodyn. 2019, 187, 48-60. [CrossRef]

18. Darwish, M.; El Damatty, A. Critical Parameters and Configurations Affecting the Analysis and Design of Guyed Transmission Towers under Downburst Loading. Pr. Period. Struct. Des. Constr. 2017, 22, 4016017. [CrossRef]

19. Liu, Z. General Design of Power Transmission and Transformation Project of State Grid Corporation of China; China Electric Power Press: Beijing, China, 2011; Volume 50.

20. Marelli, S.; Sudret, B. UQLab: A Framework for Uncertainty Quantification in Matlab. In Proceedings of the Second International Conference on Vulnerability, Risk Analysis and Management (ICVRAM2014), Liverpool, UK, 13-16 July 2014; pp. 2554-2563.

21. Santner, T.J.; Williams, B.J.; Notz, W.I. The Design and Analysis of Computer Experiments; Springer: Berlin/Heidelberg, Germany, 2003.

22. Shi, J.; Chu, L.; Braun, R. A Kriging Surrogate Model for Uncertainty Analysis of Graphene Based on a Finite Element Method. Int. J. Mol. Sci. 2019, 20, 2355. [CrossRef] [PubMed]

23. Chu, L.; Shi, J.; De Cursi, E.S. Kriging Surrogate Model for Resonance Frequency Analysis of Dental Implants by a Latin Hypercube-Based Finite Element Method. Appl. Bionics Biomech. 2019, 2019, 1-14. [CrossRef] [PubMed]

24. Lataniotis, C.; Marelli, S.; Sudret, B. UQLAB User Manual-Kriging (Gaussian Process Modelling); Report UQLab-V1.2-105; Chair of Risk, Safety \& Uncertainty Quantification, ETH Zurich: Zurich, Switzerland, 2019. 